\title{
Improving Fruit Quality and Yield of Zaghloul and Samany Date Cultivars by Spraying with Silicon Nutrient
}

"Badran, M. A. F.; Hoda S. H. Aly; O. A. Khalil and A.Y.M. Ahmed

Tropical Fruit Dep. Hort. Res. Inst., Agric. Res. Center

"E-mail: dmbadran72@yahoo.com

\begin{abstract}
The present study carried out during 2011 and 2012 seasons on "Zaghloul" and "Samany" date palm cultivars grown on new reclaimed sandy soil, at New Asuit City. Egypt, to investigate the effect of spraying with two sources of micronutrient Silicon (KSi $10 \%(\mathrm{~A} 1)$ and $\mathrm{K}_{2} \mathrm{O} 10 \%+\mathrm{SiO}_{2} 25 \%$ (A2) on fruit retention, bunch weight, total yield as well as some physical and chemical fruit properties. The two Silicon materials sprayed once, twice and thrice one week after pollination and 60 and 120 days later, respectively.

In general, inflorescences spraying with $\mathrm{A} 1$ and $\mathrm{A} 2$ sources at $0.8 \%$ one time (one week after pollination) caused a significant increase in fruit retention percentages as compared with control treatment in the two studied cultivars during both seasons. Also, extending and repeating spraying times after pollination caused a significant increase in fruit retention, bunch weight, total yield, fruit weight, fruit dimensions and fruit content of TSS, total and reducing sugars in both studied cultivars.

Palms that have been sprayed three times with $\mathrm{K}_{2} \mathrm{O} 10 \%+\mathrm{SiO}_{2} 25 \%$ (A2) produced the highest yield components (fruit retention, bunch weight and total yield), fruit weight and dimensions as compared with other treatments on both cultivars during both seasons. The chemical fruit properties (TSS \% as well as total and reducing sugars \%) under such treatments were higher than those under KSi $10 \%$ (A1) treatments in "Zaghgloul" fruits while the opposite trend observed in "Samany" fruits treated with KSi $10 \%$ (A1) where it was more effective on improving chemical fruit properties than $\mathrm{K}_{2} \mathrm{O} 10 \%+\mathrm{SiO}_{2} 25 \%$ treatments.

No significant differences were found between all silicon treatments and control on seed weight and fruit content of tannins.

Generally, the obtained results concluded that spraying "Zaghloul" and "Samany" inflorescences three times with $\mathrm{K}_{2} \mathrm{O} 10 \%+\mathrm{SiO}_{2} 25 \%$ (A2) as a source of potassium silicate was useful to gave a high yield /palm with high fruit quality and could be consider as a recommended treatment under conditions of this experiment.
\end{abstract}

Key words : Silicon, Nutrient, Zaghloul, Date Palm, Potassium Silicate

Received on: $31 / 12 / 2015$

Referees: Prof. Aiman K. Ahamed
Accepted for publication on: 19/1/2016

Prof. Farouk M. A. Mostafa 


\section{Introduction}

Date palm (Phoenix dactylifera $L)$ is the most common fruit tree grown in semi arid- regions of the Middle East and North Africa. In Egypt, According to FAO, (2010) Egypt is considered the leading country among the top ten date producers the total area devoted for date palm is 90000 feddan producing about 1,130,000 tones (Kassem, 2012). Economically "Zaghloul" and "Samany" date cultivars are the most important soft cultivars grown in Egypt; they are usually harvested and consumed at khalal stage when fruits reach at full color stage (Marzouk and Kassem, 2011).

Macro and micro elements have an important role on fruit set and retention and cause efficient yield and quality improvement (Singh and Sant Ram, 1983 and Khan et al. 1993 on mango, and Khayat et al. 2007 and El-Sabagh and Said 2012 on date palm).

Plants in the field are subjected to a large number of stresses include pests, weeds, excessive high or low temperature, soil or water salinity etc.

Silicon $(\mathrm{Si})$ has been reported to improve plant growth, increase resistance to insect attach (Ma and Takahashi 2002 and Ma. and Yamaji 2008) and enhances diseases resistance of horticultural crops (Anderson and Sosa 2001). Therefore, Si applications could improve the post harvest fruit quality of many horticultural crops not only through reducing pests and diseases incidence but also by enhancing certain fruit parameters. The most common form of Si in agriculture commodities currently used is potassium silicate $\mathrm{KSi}$, although other products, such as calcium $(\mathrm{CaSi})$ and sodium silicate $(\mathrm{NaSi})$ as well as non -tox-silicate are available. However, Si can be added as a soil application or foliar spray, depending on the form of $\mathrm{Si}$ fertilizer, potassium silicate is often applied as a foliar spray (Ma 2009).

The main aim of this study was to investigate the effects of spraying some sources of silicon micronutrient on yield and fruit quality of "Zagloul" and "Samany" date cultivars grown in new reclaimed sandy soil.

\section{Materials and Methods}

The present investigation conducted during 2011 and 2012 seasons on "Zaghloul" and "Samany" date palms (soft cultivars) grown in new reclaimed sandy soil, at private orchard located at New Assuit City. Egypt. Twenty one palms of each cultivar at full production stage were uniformly selected and received the regular cultural practices. Selected palms were moderate in pruning (8:1 leaf bunch ratio), only eight bunches of nearly equal size were left on each palm (Badran 2004). The selected bunches were pollinated by using pollen grains from the same parent in both seasons. The experiment considered a factorial experiment with two factors (2 sources of silicon (factor A) $x$ four applications of using silicon spraying (factor B) ), it was designed as compete randomized block design with three replicates (one palm for each replicate).

Spraying two sources of silicon nutrient were studied, the first source was potassium silicate $\mathrm{KSi} 10 \%$ at $0.8 \%$ (A1) formulated by central laboratory of organic agriculture 
while the second one (A2) was $\mathrm{K} 2 \mathrm{O}$ $10 \%+\mathrm{SiO} 225 \%$ at $0.8 \%$ (ELGhanem potassium silicate).

Three palms of each cultivar were treated with one of the following treatments:-

$\mathrm{T}$ 1-Control (palms sprayed with water).

$\mathrm{T}$ 2- Spraying inflorescences one time with $\mathrm{A} 1$ at one week after pollination (time1).

T 3- Spraying inflorescences two times with $\mathrm{A} 1$ at timeland 60 days later (time2).

$\mathrm{T}$ 4- Spraying inflorescences with $\mathrm{A} 1$ three times at time1, time2 and 120 days later.

$\mathrm{T}$ 5- Spraying inflorescences one time with A2 at time1.

$\mathrm{T}$ 6- Spraying inflorescences two times with $\mathrm{A} 2$ at time1 and time2.

$\mathrm{T}$ 7- Spraying inflorescences with A2 three times at time1 and time 2 and 120 days later.

After one month from pollination ten female strands per each bunch were randomly taken then fruit set $\%$ of $\mathrm{T} 1, \mathrm{~T} 2$ and $\mathrm{T} 5$ were determined according to the equation:

Number of setting fruits per strand

Fruit set $\%=$

Number of female flowers per strand

At harvest fruit retention \% were determined according to the equation:

Number of retained fruits per strand

Fruit retention $\%=$

Number of setting fruits per strand

At picking time at full color stage, all fruit bunches on each palm were harvested. Average bunch weight and total yield per palm $(\mathrm{kg})$ were recorded.

A sample of 100 fruits was taken at random from each replicate to determine the following physical and chemical characteristics

Fruit characteristics:

1-Fresh fruit and seed weight (gm)

2- Fruit height $(\mathrm{cm})$ and diameter $(\mathrm{cm})$ measured by Vernier Caliper

3- Moisture \% was determined in about $50 \mathrm{gm}$ chopped flesh sample by drying in oven at $70 \mathrm{c}$ to constant weight.

4- Total soluble solids (TSS)\% measured by a hand refractometer

5- Sugar contents including reducing and total sugars were determined according to Lane and Eynon described in A.O.A.C.( 1980)

6- Tannins content obtained according to (Winton and Winton 1958)

Data were subjected to statistical analysis according to Snedecor and Cochran (1990). The treatment means were compared using Least Significant Difference values (LSD) at $5 \%$ level.

\section{Results and Discussion}

Yield components (fruit set, fruit retention, bunch weight and total yield):

The calculated initial fruit set reached $47 . .32$ and $46.85 \%$ for Zaghloul and 45.49 and $45.18 \%$ for Samany for treatment T2 and 51.60 and $51.24 \%$ for Zaghloul and 57.49 and $55.70 \%$ for Samany for T5 comparing with control which gave 44.43 and $44.23 \%$ for Zaghloul 45.23 and $44.78 \%$ for Samany during the two seasons respectively.

The results of both seasons (Tables1 and 2) revealed that inflorescences spraying with $\mathrm{KSi} 10 \%$ (A1) or $\mathrm{K} 2 \mathrm{O} \quad 10 \%+\mathrm{SiO} 2 \quad 25 \%$ (A2) caused a significant increase in fruit retention as compared with control 
treatment in two studied cultivars during both seasons. Extending and repeating spraying times of $\mathrm{A} 1$ or $\mathrm{A} 2$ caused a significant increase in fruit retention as well as bunch weight and consequently total yield in two studied cultivars during both seasons. Hence palm yield was increased as spraying times increased. Accordingly the higher yield components (fruit retention, bunch weight and total yield) were resulted from spraying three times of A1 or A2 in both seasons.

A2 treatments were more effective as it increased all yield parameters than those of A1 treatments.
Increasing the percentages of fruit retention as well as bunch weight and total yield due to silicon spraying may be attributed to the role of silicon on improving plant growth and its ability to make balance of the nutrient uptake or the general enhancement of nutrient transport and distribution by SI (Elawad et al. 1982). Matichenkov et al. (2000) concluded that there appears to be a relationship between silicon nutrition and the health of the citrus trees. These results are in harmony with those obtained by Gad-El-Kareem (2012) and El-Wasfy (2013).

Table 1. Effect of spraying silicon on yield components (fruit retention, bunch weight and total yield) of "Zaghloul" date palm cultivar during 2011 and 2012 seasons

\begin{tabular}{|c|c|c|c|c|c|c|c|c|c|}
\hline \multirow{4}{*}{$\begin{array}{c}\text { Frequencies } \\
\text { of spraying } \\
\text { (B) }\end{array}$} & \multicolumn{3}{|c|}{ Fruit retention $\%$} & \multicolumn{3}{|c|}{ Bunch weight (kg) } & \multicolumn{3}{|c|}{ Total yield/ palm (kg) } \\
\hline & \multicolumn{9}{|c|}{2011} \\
\hline & \multicolumn{9}{|c|}{ Source of silicon $(\mathrm{A})^{*}$} \\
\hline & a 1 & a 2 & Mean & a 1 & A 2 & Mean & a 1 & a 2 & Mean \\
\hline b 0 control & 25.32 & 25.32 & 25.32 & 11.20 & 11.20 & 11.20 & 82.21 & 82.21 & 82.21 \\
\hline b 1 once & 27.42 & 30.32 & 28.87 & 11.50 & 14.39 & 12.95 & 89.29 & 100.47 & 94.88 \\
\hline b 2 twice & 29.45 & 30.18 & 29.82 & 12.57 & 14.50 & 13.54 & 96.28 & 107.16 & 101.72 \\
\hline b 3thrice & 29.53 & 32.43 & 31.13 & 12.86 & 15.22 & 14.04 & 98.23 & 110.16 & 104.20 \\
\hline Mean & 27.93 & 29.56 & & 12.03 & 13.83 & & 91.50 & 100.00 & \\
\hline L S D at $5 \%$ & $\begin{array}{c}\mathrm{A} \\
0.03\end{array}$ & $\begin{array}{c}\text { B } \\
0.05\end{array}$ & $\begin{array}{c}\text { AB } \\
0.07\end{array}$ & $\begin{array}{c}\text { A } \\
0.07\end{array}$ & $\begin{array}{c}\text { B } \\
0.10\end{array}$ & $\begin{array}{c}\mathrm{AB} \\
0.12\end{array}$ & $\begin{array}{c}\text { A } \\
0.32\end{array}$ & $\begin{array}{c}\text { B } \\
0.45\end{array}$ & $\begin{array}{c}\mathrm{AB} \\
0.62\end{array}$ \\
\hline \multicolumn{10}{|c|}{2012} \\
\hline b 0 control & 24.88 & 24.88 & 24.88 & 11.25 & 11.25 & 11.25 & 86.14 & 86.14 & 86.14 \\
\hline b 1 once & 27.02 & 31.10 & 29.06 & 12.04 & 14.88 & 13.46 & 91.52 & 102.24 & 96.88 \\
\hline b 2 twice & 29.22 & 30.45 & 29.84 & 12.50 & 15.01 & 13.76 & 95.23 & 108.20 & 101.72 \\
\hline b 3 thrice & 30.05 & 33.01 & 31.53 & 12.97 & 15.45 & 14.21 & 98.61 & 111.30 & 104.96 \\
\hline Mean & 27.79 & 29.86 & & 12.19 & 14.15 & & 92.88 & 101.97 & \\
\hline L S D at $5 \%$ & $\begin{array}{c}\mathrm{A} \\
0.45\end{array}$ & $\begin{array}{c}\mathrm{B} \\
0.62\end{array}$ & $\begin{array}{c}\mathrm{AB} \\
0.89\end{array}$ & $\begin{array}{c}\mathrm{A} \\
0.12\end{array}$ & $\begin{array}{c}\mathrm{B} \\
0.17\end{array}$ & $\begin{array}{c}\mathrm{AB} \\
0.25\end{array}$ & $\begin{array}{c}\mathrm{A} \\
1.07\end{array}$ & $\begin{array}{c}\mathrm{B} \\
1.51\end{array}$ & $\begin{array}{c}\mathrm{AB} \\
2.13\end{array}$ \\
\hline
\end{tabular}


Table 2. Effect of silicon spraying on yield components (fruit retention, bunch weight and total yield) of "Samany" date palm cultivar during 2011 and 2012 seasons

\begin{tabular}{|c|c|c|c|c|c|c|c|c|c|}
\hline \multirow{4}{*}{$\begin{array}{c}\text { Frequencies } \\
\text { of spraying } \\
\text { (B) }\end{array}$} & \multicolumn{3}{|c|}{ Fruit retention \% } & \multicolumn{3}{|c|}{ Bunch weight (kg) } & \multicolumn{3}{|c|}{ Total yield/ palm (kg) } \\
\hline & \multicolumn{9}{|c|}{2011} \\
\hline & \multicolumn{9}{|c|}{ Source of silicon $(\mathrm{A})^{*}$} \\
\hline & a 1 & a 2 & Mean & a 1 & a 2 & Mean & a 1 & a 2 & Mean \\
\hline b 0 control & 23.87 & 23.87 & 23.87 & 13.08 & 13.08 & 13.08 & 82.89 & 82.89 & 82.89 \\
\hline b 1 once & 29.25 & 32.40 & 30.83 & 14.69 & 20.44 & 17.57 & 91.00 & 128.49 & 109.75 \\
\hline b 2 twice & 30.65 & 34.31 & 32.48 & $16 . .38$ & 21.11 & 18.75 & 98.67 & 137.40 & 118.04 \\
\hline b 3 thrice & 31.40 & 37.55 & 34.48 & 18.31 & 21.76 & 20.04 & 107.61 & 143.13 & 125.37 \\
\hline Mean & 28.79 & 32.03 & & 15.62 & 19.10 & & 95.04 & 122.98 & \\
\hline L S D at $5 \%$ & $\begin{array}{c}\mathrm{A} \\
0.37\end{array}$ & $\begin{array}{c}\mathrm{B} \\
0.52\end{array}$ & $\begin{array}{c}\mathrm{AB} \\
0.74\end{array}$ & $\begin{array}{c}\mathrm{A} \\
0.03\end{array}$ & $\begin{array}{c}\mathrm{B} \\
0.05\end{array}$ & $\begin{array}{c}\text { AB } \\
0.07\end{array}$ & $\begin{array}{c}\mathrm{A} \\
2.70\end{array}$ & $\begin{array}{c}\mathrm{B} \\
3.79\end{array}$ & $\begin{array}{c}\mathrm{AB} \\
5.35\end{array}$ \\
\hline \multicolumn{10}{|c|}{2012} \\
\hline b 0 control & 23.30 & 23.30 & 23.30 & 13.97 & 13.97 & 13.97 & 90.45 & 90.45 & 90.45 \\
\hline b 1 once & 29.18 & 32.45 & 30.82 & 14.87 & 21.04 & 17.96 & 93.56 & 140.20 & 116.88 \\
\hline b 2 twice & 30.51 & 34.87 & 32.69 & 16.03 & 22.25 & 19.14 & 101.45 & 150.40 & 125.93 \\
\hline b 3 thrice & 31.00 & 37.92 & 34.46 & 17.98 & 22.89 & 20.44 & 112.70 & 157.50 & 134.95 \\
\hline Mean & 28.50 & 32.14 & & 15.71 & 20.04 & & 99.54 & 134.64 & \\
\hline L S D at $5 \%$ & $\begin{array}{c}\mathrm{A} \\
0.07\end{array}$ & $\begin{array}{c}\text { B } \\
0.10\end{array}$ & $\begin{array}{c}\mathrm{AB} \\
0.12\end{array}$ & $\begin{array}{c}\mathrm{A} \\
0.07\end{array}$ & $\begin{array}{c}\mathrm{B} \\
0.11\end{array}$ & $\begin{array}{c}\mathrm{AB} \\
0.12\end{array}$ & $\begin{array}{c}\mathrm{A} \\
1.71\end{array}$ & $\begin{array}{c}\text { B } \\
2.35\end{array}$ & $\begin{array}{c}\mathrm{AB} \\
3.30\end{array}$ \\
\hline
\end{tabular}

\section{Physical fruit characteristics}

The effect of different silicon spraying treatments on physical fruit characteristics of "Zaghloul" and "Samany" date cultivars are presented in Tables 3 and 4 . The results of both seasons revealed that spraying the inflorescences with silicon sources significantly improved fruit weight and fruit dimensions comparing with control (untreated), these differences were detected between various treatments of silicon.

Additionally, repeating spraying times of A1 or A2 caused a significant increase in fruit weight and fruit dimensions in the two studied cultivars during both seasons. Spraying with $\mathrm{A} 2\left(\mathrm{~K}_{2} \mathrm{O} 10 \%+\mathrm{SiO}_{2} 25 \%\right)$ was more effective than using A1 (KSi 10 $\%)$ on improving physical fruit characteristics of the two cultivars. Hence, the greatest fruit weight and dimension were obtained by spraying with $\mathrm{K}_{2} \mathrm{O} \quad 10 \%+\mathrm{SiO}_{2} 25 \%$ (A2) three times in both seasons.

In regard to seed weight the obtained data presented in Tables 3 and 4 showed that there were no significant differences of seed weight between different silicon treatments including the control, this was true in the two cultivars during both seasons. In other words, the increase in fruit weight by silicon treatments could be mainly attributed to the increase in flesh weight without regard to the changes of seed weight. These results are in harmony with those obtained by Gad-El-Kareem (2012) and ElWasfy (2013) who found that supplying the palms via leaves with silicon and / or royal jelly significantly improved fruit weight. Also Matichenkov et al. (2000) found a significant effect between Si application and Valencia orange fruit quality. 
Table 3. Effect of silicon spraying on some physical characteristics of "Zaghloul" dates during 2011 and 2012 season

\begin{tabular}{|c|c|c|c|c|c|c|c|c|c|c|c|c|}
\hline \multirow{4}{*}{$\begin{array}{c}\text { Frequencies } \\
\text { of spraying } \\
\text { (B) }\end{array}$} & \multicolumn{3}{|c|}{$\begin{array}{l}\text { Fruit weight } \\
\text { (g) }\end{array}$} & \multicolumn{3}{|c|}{$\begin{array}{l}\text { Fruit length } \\
(\mathrm{cm})\end{array}$} & \multicolumn{3}{|c|}{$\begin{array}{l}\text { Fruit diameter } \\
(\mathrm{cm})\end{array}$} & \multicolumn{3}{|c|}{$\begin{array}{l}\text { Seed weight } \\
\text { (g) }\end{array}$} \\
\hline & \multicolumn{12}{|c|}{2011} \\
\hline & \multicolumn{12}{|c|}{ Source of silicon ( A) } \\
\hline & a1 & a 2 & Mean & a 1 & a 2 & Mean & a 1 & a 2 & Mean & a 1 & $\mathrm{a} 2$ & Mean \\
\hline b 0 control & 15.57 & 5.57 & 15.57 & 4.72 & 4.72 & 4.72 & 1.80 & 1.80 & 1.80 & 1.09 & 1.09 & 1.09 \\
\hline b 1 once & 7.50 & .44 & 18.47 & 4.77 & 5.26 & 5 & 1.94 & 2.19 & 2.07 & 1.20 & 1.38 & 1.29 \\
\hline b 2tv & 7 & & 19.60 & 4.90 & 5.43 & & 2.02 & 3 & 3 & 1.30 & 2 & 1.36 \\
\hline b 3 thrice & .14 & 23.13 & 21.14 & 5.01 & 5.50 & 5 & 2.10 & 2.26 & 8 & 1.32 & 1.48 & 1.40 \\
\hline Mea & 17.57 & 19.82 & & 4.85 & 5.23 & & 1.97 & 2.12 & & 1.23 & 1.34 & \\
\hline \multirow{3}{*}{$\mathrm{L} \mathrm{S} \mathrm{D} \mathrm{at} 5$} & $\mathrm{~A}$ & $\mathrm{D}$ & & A & 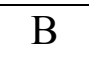 & & $\Lambda$ & 8 & & $\mathrm{~A}$ & $\mathrm{P}$ & $\overline{\mathrm{AB}}$ \\
\hline & 0.05 & 0.07 & 0 & 0.02 & 0.0 & 0.04 & 0.02 & 0.03 & 5 & 0.01 & N S & NS \\
\hline & \multicolumn{12}{|c|}{2012} \\
\hline b 0 control & 15.52 & .52 & 15.52 & 4.77 & 4.77 & 4.77 & 1.78 & 1.78 & .78 & 1.11 & 1.11 & 1.11 \\
\hline b 10 & 17.52 & 9.72 & 18.62 & 4.82 & 5.24 & 5.03 & 1.92 & 2.22 & 2.07 & 1.22 & 1.39 & 1.31 \\
\hline b 2 twice & 18.11 & 21.40 & 19.76 & 4.93 & 5.44 & 5.19 & 2.00 & 2.19 & 2.10 & 1.28 & 1.44 & 1.36 \\
\hline b 3 thrice & 19.60 & 23.45 & 21.53 & 5.10 & 5.56 & 5.33 & 2.13 & 2.31 & 2.22 & 1.33 & 1.44 & 1.39 \\
\hline Mean & 17.69 & 20.02 & & 4.91 & 5.25 & & 1.96 & 2.13 & & 1.24 & 1.35 & \\
\hline L S I & $\begin{array}{c}\mathrm{A} \\
0.09\end{array}$ & $\begin{array}{c}\text { B } \\
0.12\end{array}$ & $\begin{array}{c}\mathbf{A B} \\
0.17\end{array}$ & $\begin{array}{c}\mathrm{A} \\
0.05\end{array}$ & $\begin{array}{c}\text { B } \\
\text { 0.07 }\end{array}$ & $\begin{array}{l}\mathrm{AB} \\
\mathbf{0 . 1 0}\end{array}$ & $\begin{array}{c}A \\
0.03\end{array}$ & $\begin{array}{c}\text { B } \\
0.05\end{array}$ & $\begin{array}{l}\text { AB } \\
0.07\end{array}$ & $\begin{array}{c}\text { A } \\
\text { NS }\end{array}$ & $\begin{array}{c}\text { B } \\
\text { NS }\end{array}$ & $\begin{array}{l}\text { AB } \\
\text { NS }\end{array}$ \\
\hline
\end{tabular}

Table 4. Effect of silicon spraying on some physical characteristics of "Samany" dates during 2011 and 2012 seasons

\begin{tabular}{|c|c|c|c|c|c|c|c|c|c|c|c|c|}
\hline \multirow{4}{*}{$\begin{array}{l}\text { Frequencies } \\
\text { of spraying } \\
\text { (B) }\end{array}$} & \multicolumn{3}{|c|}{$\begin{array}{c}\text { Fruit weight } \\
(\mathrm{g})\end{array}$} & \multicolumn{3}{|c|}{$\begin{array}{c}\text { Fruit length } \\
(\mathrm{cm})\end{array}$} & \multicolumn{3}{|c|}{$\begin{array}{c}\text { Fruit diameter } \\
(\mathrm{cm})\end{array}$} & \multicolumn{3}{|c|}{$\begin{array}{l}\text { Seed weight } \\
(\mathrm{g})\end{array}$} \\
\hline & \multicolumn{12}{|c|}{2011} \\
\hline & \multicolumn{12}{|c|}{ Source of silicon ( A) } \\
\hline & a 1 & a 2 & mean & a 1 & a 2 & Mean & a 1 & a 2 & Mean & a 1 & a 2 & 10 \\
\hline b 0 con & 20.32 & 20.32 & 20.32 & 4.90 & 4.90 & 4.90 & 2.23 & 2.23 & 2.23 & 3.09 & 09 & 09 \\
\hline b 1 once & 21.94 & 24.98 & 3.46 & 5.14 & 5.77 & 5.46 & 2.27 & 2.45 & 36 & 3.17 & 3.32 & .25 \\
\hline b 2 tv & 22.03 & 27.80 & 24.92 & 5.27 & 5.78 & 5.53 & 2.38 & 2.50 & 44 & 3.19 & 41 & .30 \\
\hline b 3 thrice & 4.93 & 28.55 & 26.74 & 5.62 & 5.80 & 5.71 & 2.39 & 2.50 & 2.45 & 3.27 & 3.42 & 3.35 \\
\hline Mean & 22.31 & 25.41 & & 5.23 & 5.56 & & 2.32 & 2.42 & & 3.18 & 3.31 & \\
\hline \multirow[t]{2}{*}{ L S D at $5 \%$} & $\begin{array}{c}\mathrm{A} \\
\mathbf{0 . 0 3}\end{array}$ & $\begin{array}{c}\text { B } \\
0.05\end{array}$ & \begin{tabular}{c|} 
AB \\
0.06
\end{tabular} & $\begin{array}{c}\text { A } \\
0.02\end{array}$ & $\begin{array}{c}\text { B } \\
0.02\end{array}$ & $\begin{array}{l}\mathrm{AB} \\
0.05\end{array}$ & $\begin{array}{c}\text { A } \\
0.02\end{array}$ & $\begin{array}{c}\text { B } \\
0.02\end{array}$ & $\begin{array}{l}\text { AB } \\
0.05\end{array}$ & $\begin{array}{c}\mathbf{A} \\
0.02\end{array}$ & $\begin{array}{c}\text { B } \\
\text { NS }\end{array}$ & $\begin{array}{l}\text { AB } \\
\text { NS }\end{array}$ \\
\hline & \multicolumn{12}{|c|}{2012} \\
\hline b 0 con & 20.48 & 20.48 & 20.48 & 4.93 & 4.93 & 4.93 & 2.24 & 2.24 & 24 & 3.09 & 3.09 & 3.09 \\
\hline b 1 once & 22.01 & 25.07 & 23.54 & 5.17 & 5.82 & 5.50 & 2.29 & 2.45 & 2.37 & 3.16 & 3.33 & 3.25 \\
\hline b 2 twi & 22.65 & 27.19 & 24.92 & 5.24 & 5.90 & 5.57 & 2.36 & 2.55 & 2.46 & 3.21 & 3.44 & 3.33 \\
\hline b 3 thrice & 24.77 & 28.46 & 26.62 & 5.67 & 5.93 & 5.80 & 2.41 & 2.62 & 2.52 & 3.25 & 3.43 & 3.34 \\
\hline Mean & 22.48 & 25.30 & & 5.25 & 5.65 & & 2.33 & 2.47 & & 3.18 & 3.32 & \\
\hline S & $\begin{array}{c}\mathbf{A} \\
\mathbf{0 . 0 2}\end{array}$ & $\begin{array}{c}\text { B } \\
0.03\end{array}$ & $\begin{array}{l}\mathrm{AB} \\
\mathbf{0 . 0 4} \\
\end{array}$ & $\begin{array}{c}\text { A } \\
0.02\end{array}$ & $\begin{array}{c}\text { B } \\
0.20\end{array}$ & $\begin{array}{l}\mathrm{AB} \\
\mathbf{0 . 0 3}\end{array}$ & $\begin{array}{c}\mathrm{A} \\
\mathbf{0 . 0 3}\end{array}$ & $\begin{array}{c}\text { B } \\
0.04\end{array}$ & $\begin{array}{l}\mathbf{A B} \\
0.06\end{array}$ & $\begin{array}{c}\text { A } \\
\text { NS } \\
\end{array}$ & $\begin{array}{c}\text { B } \\
\text { NS }\end{array}$ & $\begin{array}{l}\text { AB } \\
\text { NS }\end{array}$ \\
\hline
\end{tabular}




\section{Chemical fruit characteristics:}

Tables 5 and 6 showed some fruit chemical characteristics as TSS (total soluble solids) tannins and sugar contents (reducing and total) of "Zaghloul" and "Samany" date palm cultivars as affected by spraying with two materials of silicon (A1 and A2) during the two studied seasons, 2011 and 2012. Data indicated that, total soluble solids (TSS) as well as reducing and total sugars of the two cultivars significantly increased by spraying with different silicon treatments compared with control (untreated) in both seasons. All A2 ( $\mathrm{K}_{2} \mathrm{O} 10 \%+$ $\mathrm{SiO}_{2} 25 \%$ ) treatments resulted in higher percentages of TSS, reducing and total sugars content of "Zaghloul" fruits than those of A1
(KSi $10 \%$ ) treatments while the opposite was true on "Samany" cultivar, i.e. A1 treatments were most effective on improving chemical fruit properties than all A2 treatments.

Data also revealed that the differences between silicon treatments in tannins content were not significant in both cvs during the two seasons.

The previous positive effect of the silicon sources on chemical fruit properties are due to their role on stimulating antioxidant systems in plants consequently increasing the resistance of plants to unsuitable conditions (Epsttein and Bloom, 2003). The current results are in the same line with those reported by Gad-ElKareem 2012 and El-Wasfy 2013.

Table 5. Effect of silicon spraying on some chemical characteristics of "Zaghloul" dates during 2011 and 2012 seasons

\begin{tabular}{|c|c|c|c|c|c|c|c|c|c|c|c|c|}
\hline \multirow{4}{*}{$\begin{array}{c}\text { Frequencies } \\
\text { of spraying } \\
\text { (B) }\end{array}$} & \multicolumn{3}{|c|}{ T S S \% } & \multicolumn{3}{|c|}{ Total sugars $\%$} & \multicolumn{3}{|c|}{ Reducing sugars $\%$} & \multicolumn{3}{|c|}{ Tannins $\%$} \\
\hline & \multicolumn{12}{|c|}{2011} \\
\hline & \multicolumn{12}{|c|}{ Source of silicon $(\mathrm{A})$} \\
\hline & a1 & a 2 & Mean & a 1 & a 2 & Mean & a 1 & a 2 & Mean & a 1 & a 2 & Mean \\
\hline b 0 control & 26.04 & 26.04 & 26.04 & 21.04 & 21.04 & 21.04 & 18.35 & 18.35 & 18.35 & 0.218 & 0.218 & 0.218 \\
\hline b 1 once & 27.79 & 31.06 & 29.44 & 22.34 & 28.47 & 25.41 & 19.04 & 22.47 & 20.76 & 0.203 & 0.203 & 0.203 \\
\hline b 2 twice & 28.30 & 31.47 & 29.89 & 22.36 & 29.58 & 25.97 & 19.80 & 22.05 & 20.93 & 0.205 & 0.210 & 0.208 \\
\hline b 3 thric & 2.76 & 33.34 & 33.05 & 30.18 & 31.65 & 30.92 & 23.14 & 23.26 & 23.20 & 0.205 & 0.210 & 0.208 \\
\hline Mea & 28.72 & 30.48 & & 23.98 & 27.69 & & 20.08 & 21.56 & & 0.207 & 0.210 & \\
\hline \multirow[t]{2}{*}{ L S D at $5 \%$} & $\begin{array}{c}\text { A } \\
017\end{array}$ & $\begin{array}{c}\mathrm{B} \\
0.25\end{array}$ & $\begin{array}{c}\mathrm{AB} \\
0.35\end{array}$ & \begin{tabular}{c|}
$\mathrm{A}$ \\
0.09
\end{tabular} & $\begin{array}{c}\mathrm{B} \\
0.12\end{array}$ & $\begin{array}{c}\mathrm{AB} \\
0.17\end{array}$ & $\begin{array}{c}\mathrm{A} \\
0.05\end{array}$ & $\begin{array}{c}\mathrm{B} \\
0.07\end{array}$ & $\begin{array}{l}\mathrm{AB} \\
0.12\end{array}$ & $\begin{array}{c}\mathrm{A} \\
0.01\end{array}$ & $\begin{array}{l}\mathrm{B} \\
\mathrm{NS}\end{array}$ & $\begin{array}{l}\mathrm{AB} \\
\mathrm{NS}\end{array}$ \\
\hline & \multicolumn{12}{|c|}{2012} \\
\hline b 0 control & 26.00 & 26.00 & 26.00 & 21.00 & 21.00 & 21.00 & 18.24 & 18.24 & 18.24 & 0.218 & 0.218 & 0.218 \\
\hline b 1 once & 27.65 & 31.00 & 29.33 & 22.07 & 29.04 & 25.55 & 19.20 & 22.18 & 20.69 & 0.218 & 0.213 & 0.216 \\
\hline b 2 twice & 28.09 & 31.28 & 29.69 & 22.38 & 29.65 & 26.02 & 20.02 & 21.98 & 21.00 & 0.210 & 0.203 & 0.207 \\
\hline b 3 thrice & 32.50 & 33.18 & 32.84 & 30.45 & 31.72 & 31.09 & 23.14 & 23.17 & 23.16 & 0.210 & 0.210 & 0.210 \\
\hline Mean & 28.56 & 30.37 & & 23.98 & 27.85 & & 20.15 & 21.39 & & 0.214 & 0.211 & \\
\hline L S D at $5 \%$ & $\begin{array}{c}\mathrm{A} \\
0.37 \\
\end{array}$ & $\begin{array}{c}\mathrm{B} \\
0.52 \\
\end{array}$ & $\begin{array}{c}\mathrm{AB} \\
0.74 \\
\end{array}$ & $\begin{array}{c}\mathrm{A} \\
0.80 \\
\end{array}$ & $\begin{array}{c}\mathrm{B} \\
1.10 \\
\end{array}$ & $\begin{array}{c}\mathrm{AB} \\
1.60 \\
\end{array}$ & $\begin{array}{c}\text { A } \\
0.25 \\
\end{array}$ & $\begin{array}{c}\mathrm{B} \\
0.35 \\
\end{array}$ & $\begin{array}{c}\mathrm{AB} \\
0.50 \\
\end{array}$ & $\begin{array}{c}\text { A } \\
\text { NS } \\
\end{array}$ & $\begin{array}{c}\text { B } \\
\text { NS } \\
\end{array}$ & $\begin{array}{l}\mathrm{AB} \\
\mathrm{NS} \\
\end{array}$ \\
\hline
\end{tabular}


Table 6. Effect of silicon spraying on some chemical characteristics of "Samany" dates during 2011 and 2012 seasons

\begin{tabular}{|c|c|c|c|c|c|c|c|c|c|c|c|c|}
\hline \multirow{4}{*}{$\begin{array}{l}\text { Frequencies } \\
\text { of spraying } \\
\text { (B) }\end{array}$} & \multicolumn{3}{|c|}{ T S S \% } & \multicolumn{3}{|c|}{ Total sugars $\%$} & \multicolumn{3}{|c|}{ Reducing sugars $\%$} & \multicolumn{3}{|c|}{ Tannins $\%$} \\
\hline & \multicolumn{12}{|c|}{2011} \\
\hline & \multicolumn{12}{|c|}{ Source of silicon (A) } \\
\hline & a1 & a 2 & Mean & a 1 & a 2 & Mean & a 1 & a 2 & Mean & a 1 & a 2 & Mean \\
\hline $0 \mathrm{Oc}$ & 33.26 & 32.26 & 26 & 29.49 & 29.49 & 9 & 23.61 & 23.61 & 23.61 & 0.178 & 178 & 0.178 \\
\hline b 1 & & 35.30 & 35.74 & .02 & 31.50 & 1.76 & 26.00 & 24.85 & 25.43 & 154 & 178 & 0.166 \\
\hline b 21 & .28 & 35.59 & 36.94 & 34.19 & 32.48 & 33.34 & 27.29 & 25.35 & 26.32 & 0.203 & 0.201 & 0.202 \\
\hline b $3 \mathrm{t}$ & .18 & 39.09 & & 37.38 & 36.33 & & 29.39 & 28.09 & 28 & 0.203 & 0.203 & 0.20 \\
\hline Mear & 36.98 & 35.56 & & 33.27 & 32.45 & & 26.57 & 25.48 & & 0.185 & 0.190 & 0.188 \\
\hline \multirow[t]{2}{*}{ L S D at $5 \%$} & $\begin{array}{c}\text { A } \\
0.06\end{array}$ & $\begin{array}{c}\text { B } \\
0.09\end{array}$ & & $\begin{array}{c}\text { A } \\
0.01\end{array}$ & $\begin{array}{c}\mathrm{B} \\
0.02\end{array}$ & $\begin{array}{c}\mathrm{AB} \\
0.03\end{array}$ & $\begin{array}{c}\mathrm{A} \\
0.04\end{array}$ & $\begin{array}{c}\mathrm{B} \\
0.05\end{array}$ & $\begin{array}{c}\mathrm{AB} \\
0.07\end{array}$ & $\begin{array}{c}\mathrm{A} \\
0.01\end{array}$ & $\begin{array}{c}\mathrm{B} \\
\mathrm{NS}\end{array}$ & $\begin{array}{l}\mathrm{AB} \\
\mathrm{NS}\end{array}$ \\
\hline & \multicolumn{12}{|c|}{2012} \\
\hline $\mathrm{b} 0 \mathrm{c}$ & & 33.28 & 33.28 & 29.19 & 29.19 & 29.19 & 22.45 & 22.45 & 22.45 & 0.178 & 178 & 0.178 \\
\hline b 10 & 36.12 & 34.75 & 35.44 & 32.00 & 31.47 & 31.74 & 25.89 & 24.19 & 25.04 & 0.154 & 0.201 & 0.178 \\
\hline b 2 t & 37.98 & 35.09 & 36.54 & 34.72 & 32.87 & 33.80 & 27.45 & 25.39 & 26.42 & 0.154 & 0.178 & 0.166 \\
\hline b 3 tl & 39.85 & 38.79 & 39.32 & 37.56 & 35.45 & 36.51 & 30.45 & 28.16 & 29.31 & 0.201 & 0.187 & 0.194 \\
\hline Mean & 36.81 & 35.48 & & 33.37 & 32.25 & & 26.56 & 25.48 & & 0.172 & 0.186 & \\
\hline L S D at $5 \%$ & $\begin{array}{c}\text { A } \\
0.08\end{array}$ & $\begin{array}{c}\mathrm{B} \\
0.11\end{array}$ & $\begin{array}{c}\mathrm{AB} \\
0.15\end{array}$ & $\begin{array}{c}\mathrm{A} \\
0.05\end{array}$ & $\begin{array}{c}\text { B } \\
0.08\end{array}$ & $\begin{array}{l}\mathrm{AB} \\
0.11\end{array}$ & $\begin{array}{c}A \\
0.05\end{array}$ & $\begin{array}{c}\text { B } \\
0.07\end{array}$ & $\begin{array}{c}\mathrm{AB} \\
0.10\end{array}$ & $\begin{array}{l}\text { A } \\
\text { NS }\end{array}$ & $\begin{array}{c}\text { B } \\
\text { NS }\end{array}$ & $\begin{array}{l}\mathrm{AB} \\
\mathrm{NS}\end{array}$ \\
\hline
\end{tabular}

Conclusively, the obtained results give basis to conclude that, Spraying "Zaghloul" and "Samany" date palm inflorescences grown in new reclaimed soil with potassium silicate (KSi $10 \%$ ) at $0.8 \%$ or $\mathrm{K} 2 \mathrm{O}$ $10 \%+\mathrm{SiO} 225 \%$ at $0.8 \%$ had a positive effect on fruit set, yield and fruit quality. The most beneficial treatment in this concern is spraying with $\mathrm{K}_{2} \mathrm{O}$ $10 \%+\mathrm{SiO}_{2} 25 \%$ at $0.8 \%$ three times (one week after pollination, 60 days later and 120 days later) which gave a reasonable yield and high fruit weight and dimensions with high content of TSS and sugars and could be considered as a recommended treatment under the conditions of this experiment. Refernces

Anderson D.L. and Sosa O Jr. (2001): Effect of silicon on expression of resistance to sugarcane borer (Diatraea saccharalis). Proc. J. Am Soc Cane Technol., 21, 43-
50.

Association of official chemistis (1980) Official methods of analysis, A. O. A. C. 14 th. Ed. PP. 494-500 Benjamine, Franklien Station Washington D. C., U. S. A.

Badran A. M. F. (2004): Physiological studies on fruit set and yield of Zaghloul and Samany date cvs. Under Aswan conditions ph.D. Horti. Dep. Assuit Univ. (188).

Elawad S.H., Gascho G.J. and Street J.J. (1982): Response of sugarcane to silicate source and rate I: Growth and Yield", Agronomy Journal, 74, 481-484.

El-Sabagh, A. and Said (2012): Effect of bunches spraying with micro and macro nutrient on fruit retention and physical characteristics of "Deglet Noor" date cultivar during kimir stage. Res. J. 
of aric. And bio. Sci. 8 (2) 138 $: 146$.

Epstein, E. and Bloom, A. J. (2003): Mineral Nutrition of Plant, Principles and Perspectives. 2nd Ed. John Wiley \& Sons, New York pp $1-120$.

Gad El- Kareem, M. R. (2012): Improving productivity of Taimour mango trees by using glutathione, silicon and vitamins B. Minia J. of Agric. Res. \& Develop, Vol (32), No. 4 p 402 430.

Kassem H. A. (2012): The response of date palm to calcareous soil fertilization. Journal of soil Sci. and nutrition, (1):45-58.

Khan, N., A. B. Malik, M. I. Makbdoom and A.Hag, (1993): Investigations on the efficiency of exogenous synthetic growth regulators on fruit drop in mango (Mangifera indica L.). Egypt, J. Hort., 20:1-14.

Khayate M., ETafazoli, S. Eshghi and S. Rajaee (2007): effect of Nitrogen, Boron, Potassium and Zinc sprays on yield and fruit quality of date palm. americanEurasian J. Agric. \& Environ. Sci.,(2): 289-296.

Ma, J.F. (2004):"Role of silicon in enhancing the resistance of plants to biotic and abiotic stresses", Soil Sci. Plant Nutr., 50, 11-18.

Ma J.F. (2009): Silicon uptake and translocation in plants", The proceedings of the International Plant Nutrition Colloquium
XVI, Department of Plant Sciences, UC Davis'

Ma, J. F. and Takahashi, E. (2002): soil, fertilizer and plant. Silicon Research in Japan, Elsevier 1281.

MA J. F. and YAMAJI, N. (2008). Functions and transport of silicon in plants. Cell. Mol. Life, Sci. 65: 3049-3057.

Marzouk H. A. and H. A. Kassem (2011) improving fruit quality, nutritional value and yield of Zaghloul dates by the application of organic and or mineral fertilizers. Scientific horticulturae 127(2011) 249-254.

Matichenkov, V. V.; Calvert, D. V. and Snyder, G. H. (2000): Prospective of silicon fertilization for citrus in Florida. Proc. Soil and Crop Sci. Soc. of Florida 5 p: $137-141$.

El- Wasfy, M (2013): Response of Sakkoti Date Palms to Foliar Application of Royal Jelly, Silicon and Vitamins B. journal of American science 2013 ; 9 (5).

Singh, R. S. and Sant Ram (1983): Studies on the use of palm growth substances for fruit retention in mango cv. Dashehair. Ind. J. Hort. 40 :188-194.

Snedecor, C.W. and W.G. Cochran (1990). Statistical methods $7^{\text {th }}$ ed. The Iowa State Univ. Press. Ames Iowa. USA. P. 593.

Winton A. L. and K. B. Winton (1958): the analysis of food. John and sons Increased. London. Pp 853-867. 
تحسين جودة ومحصول صنفي نخيل البلح الزغلول و السمانى باستخدام الرش بعنصر

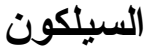

محمد احمد فواد باران ، هدى سعد حسن على ، عمر عبد الحارس خليل واحمد يسن محمد احمد ،

مركز البحوث الزر اعية ، معهد بحوث البساتين ، قسم بحوث علث الفاكهة الاستو ائية

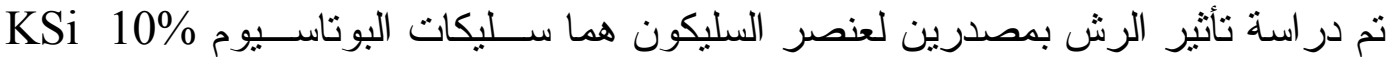

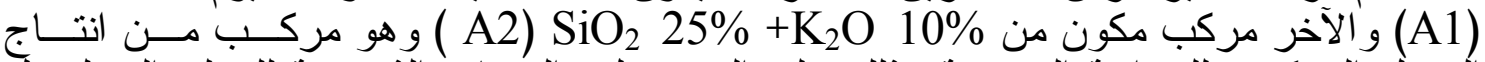

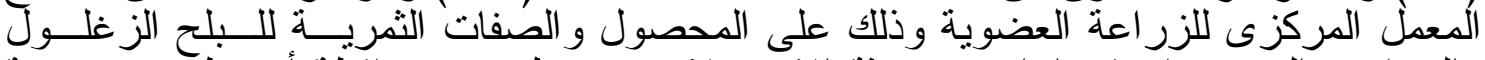

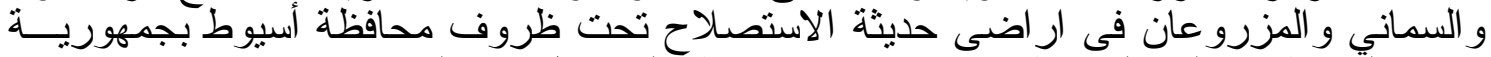

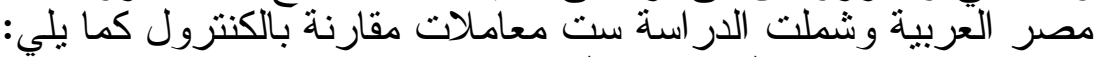

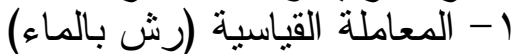

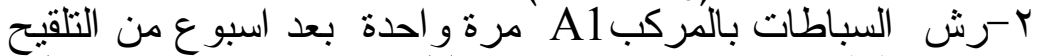

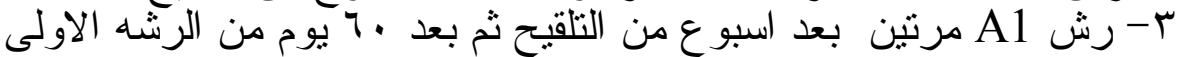

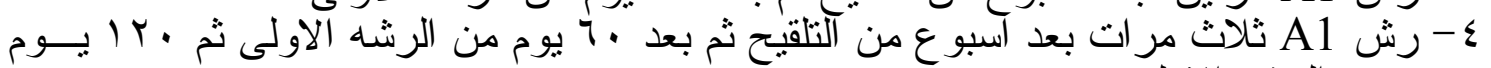

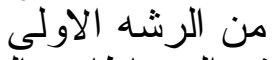
0- رش السوباطات بالمركب A2 مرة و واحدة بعد اسبوع من من التلقيح

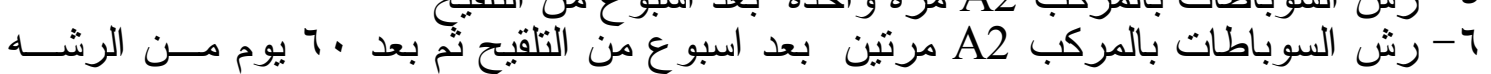

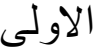

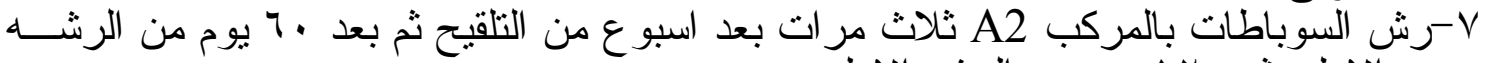

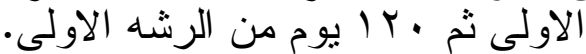

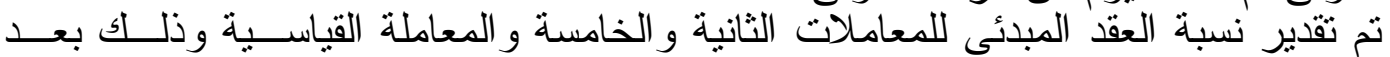

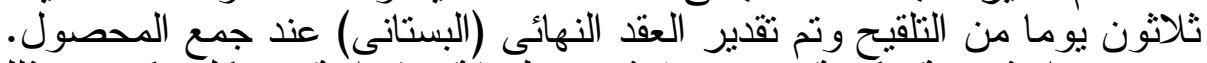

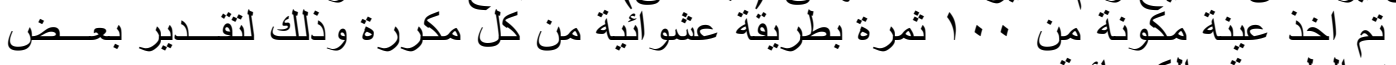
الصفات الطبيعية و الكيميائية. اولا: الصفات الطبيعية: وكانت وزن و أبعاد ( الطول و القطر ) الثمرة وكذلك وزن البذرة. ثانيا: الصفات الكيميائية:

وكانت نسبة المو اد الصلبة الذائبة و المحتوى من الــسكريات المختزلـــة و الكليـــة بجانــبـ المحتوى من التانينات وكانت ابرز النتائج المتحصل عليها كما يلي:

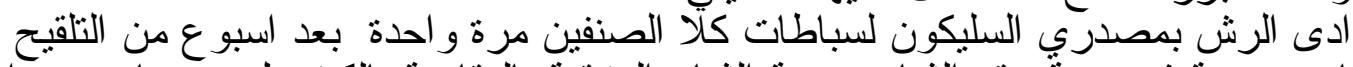

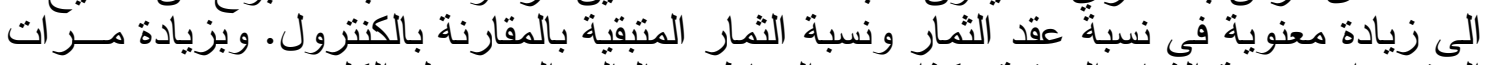

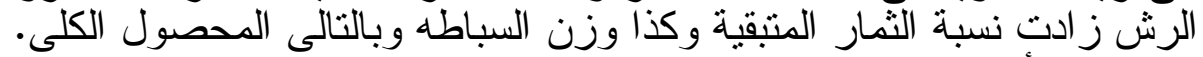

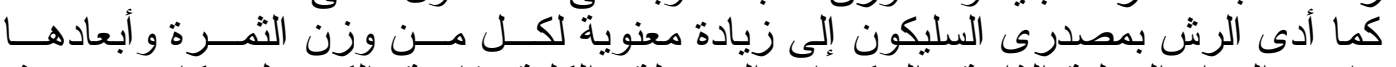

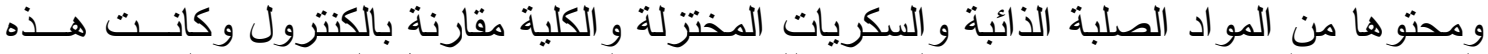

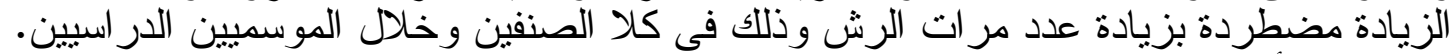

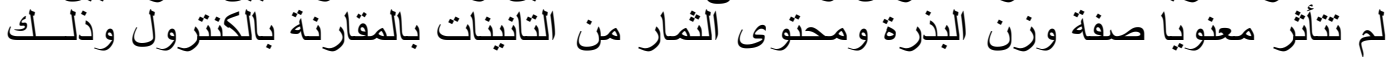

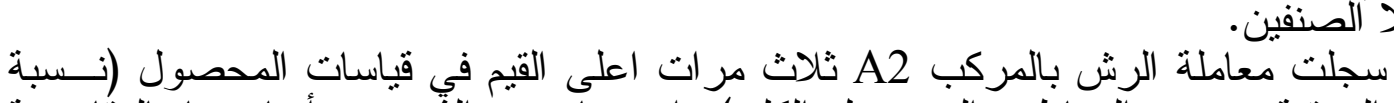

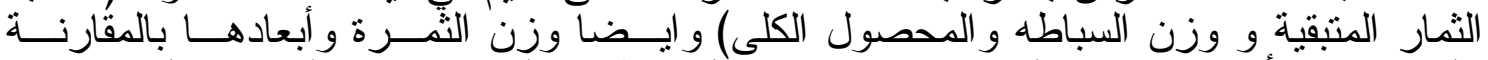

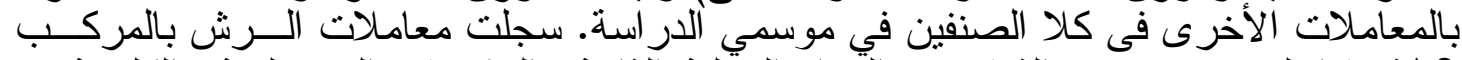

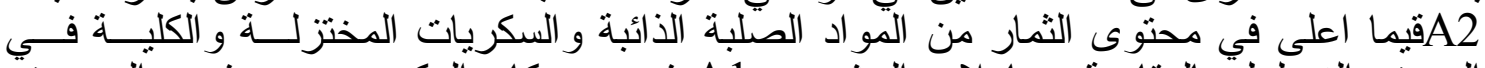

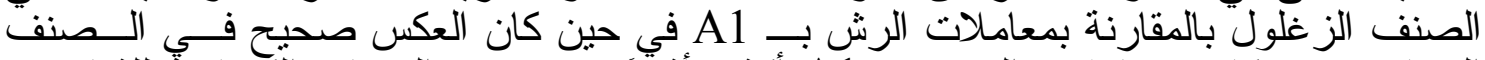

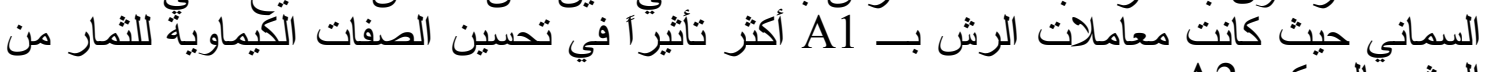

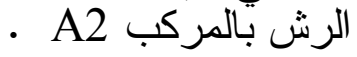




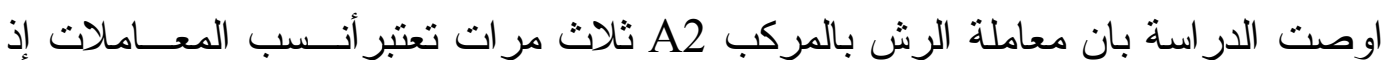

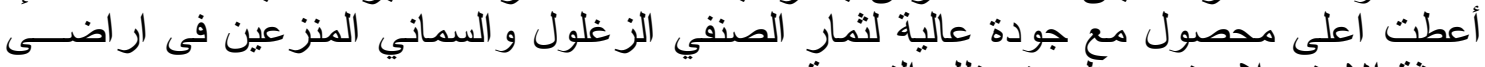
حديثة الاستصلاح تحت ظروف تلأك التجربة. 